\title{
A Large Palatal Swelling - Not Just a Dental Abscess
}

\author{
Nayan Patel ${ }^{1}$, Arif Razzak ${ }^{2, *}$ and Brian Castling ${ }^{3}$ \\ ${ }^{1}$ BDS, MFDS RCDEd [Oral and Maxillofacial Surgery DCT1, Shrewsbury and Telford Hospital NHS Trust], UK \\ ${ }^{2}$ BDS, MFDS RCSE, PGDip MedEd [Oral and Maxillofacial Surgery Speciality Doctor, Shrewsbury and Telford Hospital NHS Trust], UK \\ ${ }^{3}$ FDSRCS (Eng) FRCS (OMFS) [Oral and Maxillofacial Surgery Consultant, Shrewsbury and Telford Hospital NHS Trust], UK
}

${ }^{*}$ Corresponding author: Arif Razzak, BDS, MFDS RCSE, PGDip MedEd [Oral and Maxillofacial Surgery Speciality Doctor, Shrewsbury and Telford Hospital NHS Trust], UK; E-mail: arif.razzak1@nhs.net

Received: November 10, 2020; Accepted: November 20, 2020; Published: November 26, 2020

\begin{abstract}
Patients routinely present with intra oral swellings and commonly these are caused by dental abscesses. This case however, highlights the importance of having a differential diagnosis, and the pitfalls of managing such lesions, especially during the COVID-19 pandemic.

This report discusses a 34-year-old gentleman who presented in the Emergency Department with an intra oral swelling which appeared to be odontogenic in origin. Initially diagnosed as a dental abscess from an upper posterior tooth which was incised and drained, it was later found that the swelling was caused from cystic pathology associated with a root treated upper anterior tooth. His delayed management was due to poor attendance in primary care and this case will highlight the clinical features of his presentation and the appropriate treatment moving forward.

Clinical Relevance: To make the general dental practitioner (GDP) aware those intra oral swellings can have a multifactorial aetiology and a thorough examination is required to ascertain a definitive diagnosis and prompt referral where necessary, in order to plan the correct treatment successfully. This patient was an irregular attender in primary care, thus compromising his clinical management.
\end{abstract}

Objective Statement: To demonstrate how a common complaint must be investigated thoroughly in order to achieve a successful outcome.

\section{Introduction}

Intraoral swellings are a clinical presentation of a wide range of pathology, and clinicians should use a diagnostic sieve to rule out possible causes (Figure 1). Often intra-oral swellings can be associated with a non-vital tooth, which can cause a periapical abscess, whereby bacteria and their associated toxins spread through the apical foramen of a tooth and cause abscess formation [1]. Chronic infection in the apical tissues can initiate the development of an inflammatory cyst.

A cyst is a pathological cavity with fluid or semi-fluid contents lined by epithelium. Epithelial odontogenic cysts can be classified as

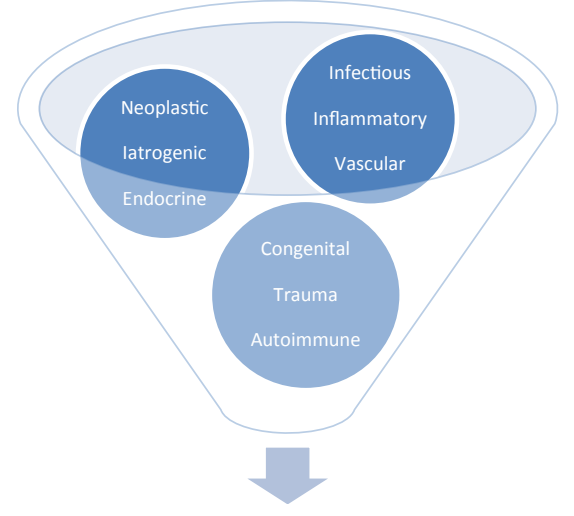

Figure 1: Diagnostic sieve showing potential aetiology of intra oral swellings. developmental or inflammatory in origin depending on where they have been derived from, however the broad discussion of these is not within the scope of this paper. Figure 2 shows a brief overview of epithelial derived odontogenic cysts.

A radicular cyst arises from longstanding chronic apical infection in a non-vital tooth which stimulates the proliferation of epithelial cell remnants. These originate from the root sheath of Hertwig and are known as the cell rests of Malassez [2]. Radicular cysts are the most common odontogenic cyst in the head and neck and $60 \%$ present in the maxilla [3]. They can be found at the apex of the tooth or originate laterally from accessory root canals.

Cysts can either be sterile or become secondarily infected and this can cause both a diagnostic and management challenge.

\section{Case Presentation}

A 34-year-old gentleman presented in the Emergency Department at the Princess Royal Hospital in Telford. He was originally due for surgical drainage of a pilonidal abscess in a sister hospital however this was postponed due to his presenting complaint of an intraoral swelling. He was consequently referred to the oral and maxillofacial surgery team.

He reported a 7-year history of recurrent mouth abscesses and dental pain, with the most recent presentation persisting for 3 months. He complained of an intermittent bilateral palatal swelling which felt 


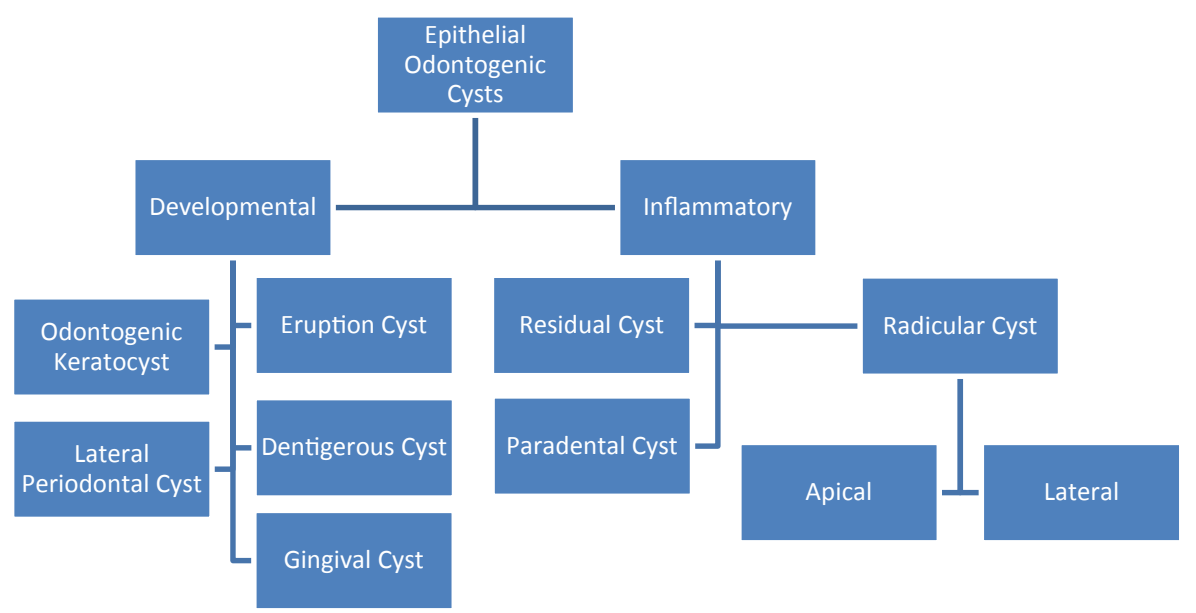

Figure 2: Classification of common epithelial odontogenic cysts.

more right sided on the day of presentation. He also complained of anosmia after a head injury 5 years ago and difficulty breathing through his right nostril as his sinuses felt 'constantly congested.' He had previously drained the swelling himself on the left side of his palate with a Stanley knife.

Medically he was fit and well and had no known drug allergies. He smokes 20 cigarettes daily and drinks roughly 16 units of alcohol a week, and at the time of presentation, he did not have access to a general dental practitioner (GDP).

\section{Initial Examination}

On examination, his $\mathrm{ABCDE}$ assessment showed no abnormalities, he had stable observations, and an unremarkable extra-oral examination.

Intraoral examination demonstrated a generalised neglected and partially restored dentition. There was a fluctuant buccal swelling adjacent to the UR6 and a separate diffuse, firm unilateral palatal swelling extending posteriorly up to the soft palate on the right side. There was no draining sinus intra orally, but he had previously recalled the taste of pus and blood. No teeth were tender to percussion or mobile.

An orthopantomogram (OPG) was requested and can be seen in Figure 3. The OPG shows a moderately restored dentition, gross caries affecting multiple teeth and generalised horizontal bone loss. The UR2 has a suboptimal root filling which appears short of the apex and has associated periapical radiolucency. Readers are advised to see how sometimes a large cystic lesion in this area can present as a pneumatised sinus on an OPG, and due to the presence of the lesion over the maxillary sinus on the right hand side, a clear diagnostic view on the likely size of this lesion was challenging by plain film radiography alone.

\section{Management}

Although the patient had presented with what seemed to be a chronic lesion, the acute swelling had to be managed initially. Under local anaesthesia, the fluctuant buccal swelling adjacent to the UR6 was incised and drained. Pus and blood exuded from the swelling

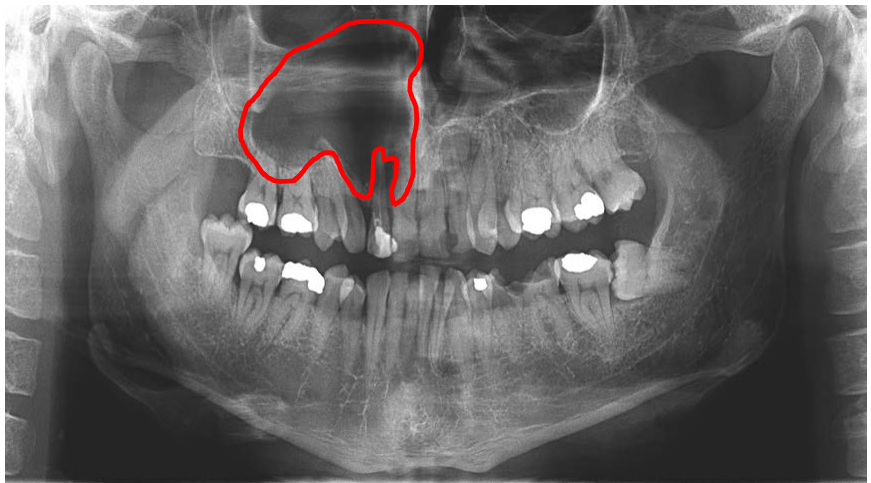

Figure 3: OPG taken on initial presentation highlighting pathology in the right maxillary antrum.

and the patient noticed a relief in pressure in the area. The unilateral right sided palatal swelling remained mobile and intact. A course of antibiotics was prescribed and post-operative instructions were given. The patient was booked for follow up and further diagnostic imaging was requested.

A follow up computed tomography (CT) scan of the sinuses was arranged in order to assess the extent and likely cause of the lesion. The CT scan reported a large cystic unilocular lesion arising from the right maxillary alveolus, likely to be of dental origin. It showed marked superior invagination and expansion of the right maxillary sinus and a defect in the hard palate. Superiorly, a wafer-thin cortex of bone separated the lesion from the ethmoidal sinus. The lesion measures $5 \mathrm{~cm}$ anterior-posteriorly, $4 \mathrm{~cm}$ medial-laterally and $4.3 \mathrm{~cm}$ craniocaudally (head to toe).

Figure 4 shows a coronal view of the lesion at its greatest width, occupying the nasal cavity on the right side. Comparing this to the left side, the lesion is clearly showing its expansion into the right inferior turbinate. Figures 5-8 demonstrate the shape and size of the lesion moving coronally in the axial view. Figure 5 demonstrates the relationship of thelesion with the UR 2 and Figure 6 shows how thelesion extended buccally adjacent to the UR6. This explains the presentation of a buccal abscess adjacent to UR6, the palatal swelling, the anosmia and the history of right sided sinus congestion. Odontogenic disease 


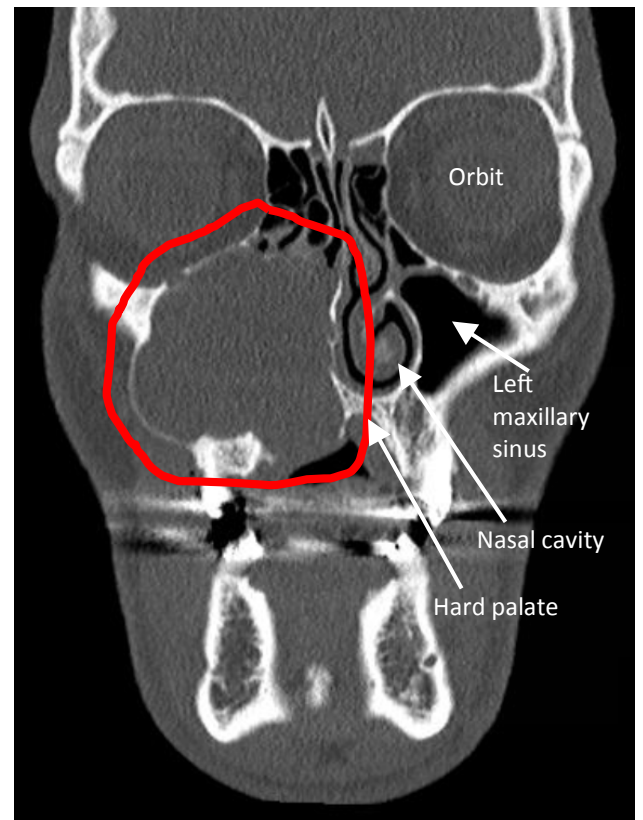

Figure 4: Coronal cross-sectional view highlighting the radicular cyst occupying right nasal cavity and inferior turbinate.

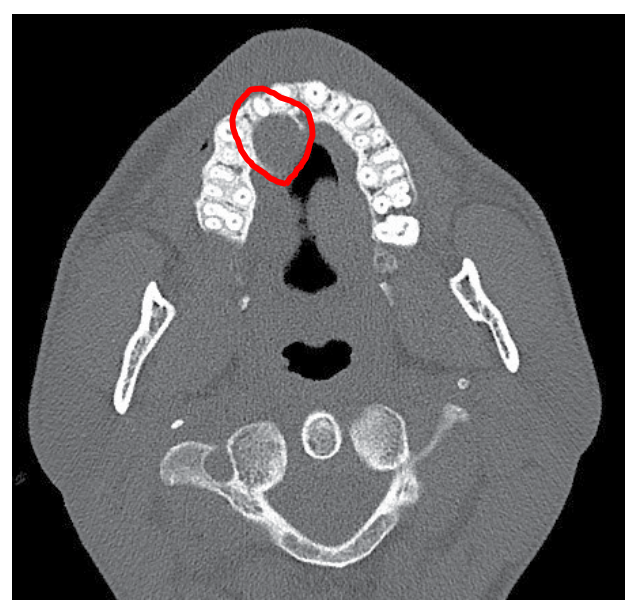

Figure 5: Axial cross-sectional view highlighting the radicular cyst arising from UR2 tooth growing palatally.

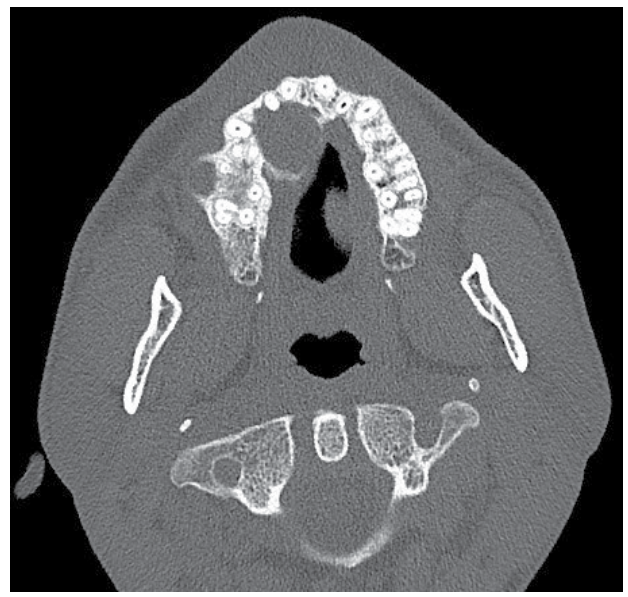

Figure 6: Axial cross-sectional view highlighting the radicular cyst growing buccally adjacent to UR6 tooth.

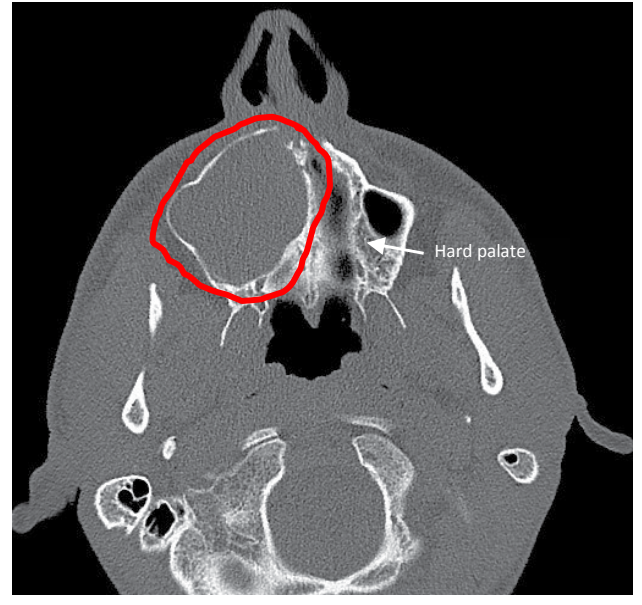

Figure 7: Axial cross-sectional view highlighting the radicular cyst invading the right hard palate.

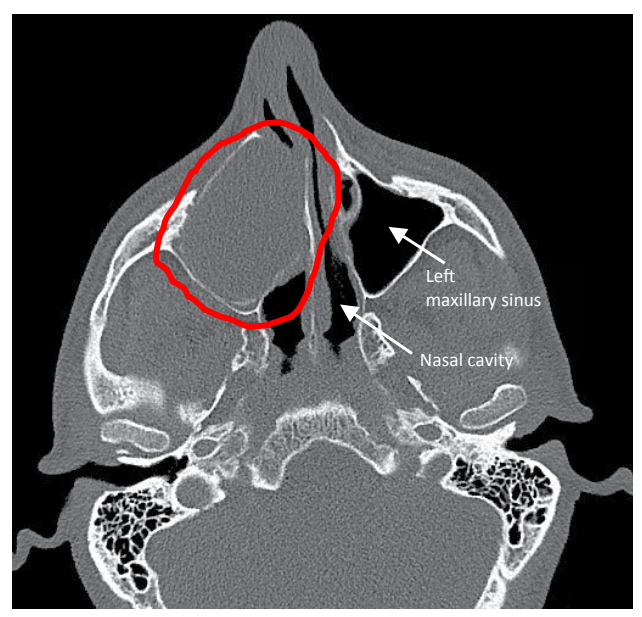

Figure 8: Axial cross-sectional view highlighting the radicular cyst occupying right nasal cavity.

is thought to be responsible for $10-12 \%$ of maxillary sinusitis cases and therefore clinicians should always consider this possibility when a patient reports sinus like symptoms [4].

After discussion with the patient, he agreed to a general anaesthetic procedure for biopsy and marsupialisation of the suspected cyst. Under general anaesthetic, the opportunity was used to remove other teeth of a poor long-term prognosis, namely the UL2 and LL56. The UR2 was also removed as part of the process of marsupialisation of the cyst.

A large buccal flap was raised and the bone overlying the cyst was carefully removed to enable adequate access. A biopsy of cyst lining was taken and the cyst underwent marsupialisation, whereby the cyst lining was sutured to form a continuous layer with the buccal mucosa (Figure 9). Upon discharge, the patient was given smoking cessation advice, instructions on regular saline irrigation to the area with the use of a monojet syringe and a course of oral antibiotics.

Due to the size of this lesion, it was felt prudent to obtain a good quality histological sample prior to attempting enucleation. Although the CT scan and OPG appeared to show that the lesion was associated with the UR2, an odontogenic keratocyst could not completely be 

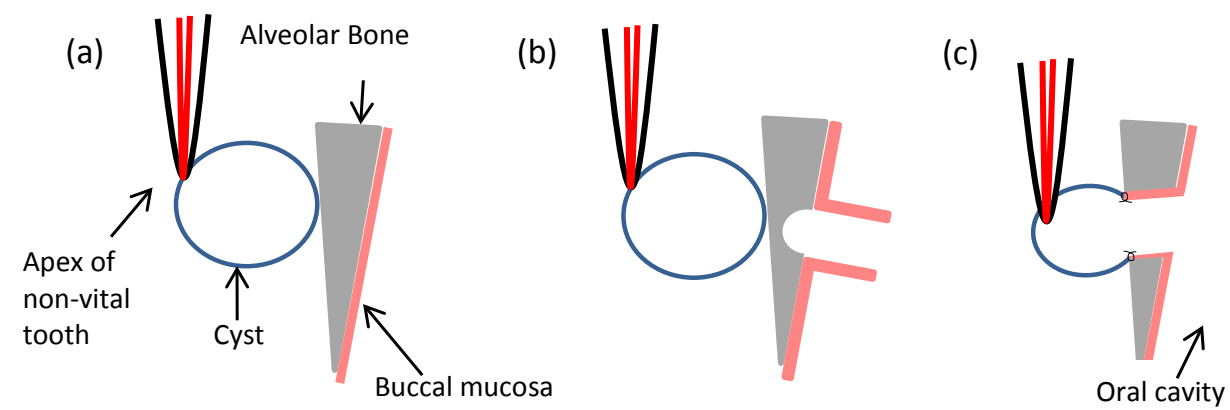

Figure 9: Marsupialisation of a cyst. (a) Pre-operative view of cyst originating from non-vital tooth. (b) Flap raised with buccal bone removal (with round bur shown) to gain access to cyst lining. (c) Cyst opened, drained and lining sutured to the oral mucosa. This prevents an osmotic pressure gradient forming, allowing the cyst to reduce in size prior to enucleation.

ruled out. As the enucleation of an odontogenic keratocyst is more involved (often involving the use of Carnoy's solution due to the cysts higher recurrence rate of up to $60 \%$ [5]), a definitive diagnosis was sought prior to a planned enucleation.

The histology showed a fibrous walled cyst lined by a hyperplastic squamous epithelium showing severe active chronic inflammation including hemosiderin - laden macrophages. These findings were consistent with the clinical impression of a radicular cyst.

The planned management for this patient was a follow up assessment of the lesion both clinically and radiographically. Once the cyst had reduced in size as a result of the marsupialisation, an enucleation was planned with a possible Caldwell-Luc approach. By carrying out an initial marsupialisation, any reduction in cyst size will have fewer profound complications compared to an enucleation at the same time of initial biopsy. At the time of writing this paper, the patient is currently due for follow up post marsupialisation, however due to the COVID-19 pandemic, this has been delayed.

\section{Aetiology}

The pathogenesis of a radicular cyst is a direct sequel to an apical granuloma. This is a sequel to chronic apical periodontitis which results in inflammatory cell, granulation and scar tissue formation in the periradicular tissues of a non-vital tooth. It is important to note that a granuloma need not always develop into a radicular cyst [6].

The development of a radicular cyst can be broken down into three phases; cyst initiation, cyst formation and cyst enlargement $[2,6]$.

\section{Cyst Initiation}

As a result of the chronic inflammatory processes at the apex of the non-vital tooth, the dormant epithelial cell rests of Malassez derived from the root sheath of Hertwig, begin to proliferate. This proliferation is influenced by bacterial endotoxins, epidermal growth factors and cytokines released by various cells in the periapical lesion $[2,6,7]$.

\section{Cyst Formation}

It is believed that the growth of these epithelial cells reaches a critical point where central cells are starved from their source of nutrition and undergo necrosis and liquefactive generation. These microcavities containing degenerative epithelial cells and tissue fluid coalesce to form a cyst cavity lined by stratified epithelium $[2,6,7]$.

\section{Cyst Enlargement}

The exact mechanism of cyst growth is not fully understood however it is generally believed to be linked to osmosis. The necrosis of central cells and lytic breakdown products increases the osmotic pressure within the cyst compared to the surrounding stroma. This gradient draws fluid into the cyst via osmosis and increases the hydrostatic pressure. The volume expansion causes peripheral epithelial cell growth in order to maintain the cyst lining. Continuous shedding of central cells maintains an osmotic gradient causing further cyst growth, bony expansion, bone resorption and cortical thinning [8]. The average rate of expansion is thought to be roughly 5 mm per year [7].

In this case, it is likely that the cyst has been present for many years, given the history of the patient being told by his GDP "many years ago" that the UR2 tooth was of a poor long-term prognosis and should be removed.

\section{Relevance for General Dental Practitioners (GDPs)}

The case discussed shows the importance of a thorough history, clinical and radiographic examination of any patient that presents with an intra-oral swelling. The phrase 'common things occur commonly' often comes to mind however GDPs should not disregard uncommon and rare diagnoses which may present initially in primary care. The duration of a presenting complaint may guide diagnosis. For example, a buccal abscess may present as a two-week history of swelling whereas a large radicular cyst may present as a seven-year history of swelling.

Taking appropriate imaging is vital in achieving an accurate diagnosis and clinicians should prompt referral where necessary. It is important to note that these lesions cannot be definitively diagnosed without biopsy and histology testing.

As a rough rule, ovoid and well circumscribed periapical radiolucencies greater than $1-1.5 \mathrm{~cm}$ in diameter may be indicative of a cyst or other pathology $[9,10]$. If an extraction is carried out and the cyst lining is not removed, it has the potential to remain (as a residual cyst) and continue to expand. As a result, extraction sockets should carefully be curetted to remove any granulation tissue or cyst lining that may be present.

Another treatment option to consider for these lesions is an apicectomy with removal and curettage of the associated cyst, 
provided that the tooth has a sound root canal treatment and coronal restoration. This is however dependent on the extent of the apical pathology.

Depending on the size of the radiolucent area radiographically, referral may be indicated.

Timely referral to secondary care in cases like these drastically improves patient outcomes and their overall treatment experience. In the above case, it is likely that the cyst has been present for some time, and unfortunately although the patient was told by his GDP when the lesion was possibly a lot more manageable, the patient reported moving geographic location and had not sought dental treatment for many years. This likely allowed for continued enlargement and expansion of the cyst. These can often remain asymptomatic until complications arise.

\section{Conclusion}

The majority of intra oral swellings are managed effectively in primary care however at times, further investigation and referral to secondary care or specialist services is required. The case discussed shows how thorough examination and accurate diagnosis are vital to allow effective management of these more complicated cases. It is thought that over $40 \%$ of periapical radiolucencies are cystic [7] and it is imperative the GDP manages these effectively as patients will routinely present to them for first line treatment in an emergency scenario.

\section{Reference}

1. Siqueira JF, Rôças IN (2013) Microbiology and Treatment of Acute Apical Abscesses. Clin Microbiol Rev 26: 255-273. [crossref]

2. Ward JP, Magar V, Franks SJ, Landini G (2004) A mathematical model of the dynamics of odontogenic cyst growth. Anal Quant Cytol Histol 26: 39-46. [crossref]

3. Narula H, Ahuja B, Yeruli R, Baliga S, Munshi AK (2011) Conservative non-surgical management of an infected radicular cyst. Contemp Clin Dent 2: 368-371. [crossref]

4. Chapman MN, Nadgir RN, Akman AS et al. (2013) Periapical Lucency around the Tooth: Radiologic Evaluation and Differential Diagnosis. Radiographics 33: 15-32. [crossref]

5. Fidele NB, Yueyu Z, Zhao Y, W Tianfu, J Liu et al. (2019) Recurrence of odontogenic keratocysts and possible prognostic factors: Review of 455 patients. Med Oral Patol Oral Cir Bucal 24(4): 491-501. [crossref]

6. Latoo S, Shah A, Jan S, et al. (2009) Radicular cyst. JK Science 11: 187-189.

7. Soames JV, Southam JC (2005) Oral Pathology (Fourth Edition). New York: Oxford University Press.

8. Cawson RA, Odell EW (2008) Cawson's Essentials of Oral Pathology and Oral Medicine (Eighth Edition). Churchill Livingstone Elsevier.

9. Scholl RJ, Kellet HM, Neumann DP, Lurie AG (1991) Cysts and Cystic Lesions of the Mandible: Clinical and Radiologic-Histopathologic Review. Radiographic 19: 11071124. [crossref]

10. Dunfee BL, Sakai O, Pistey R, Gohel A (2006) Radiologic and Pathologic Characteristics of Benign and Malignant Lesions of the Mandible. Radiographics 26: 1751-1768. [crossref]

\section{Citation:}

Nayan Patel, Arif Razzak, Brian Castling (2020) A Large Palatal Swelling - Not Just a Dental Abscess. J Dent Maxillofacial Res Volume $3(4)$ : 1-5. 